\title{
Alkaloids from the Malayan Hunteria zeylanica Gard.
}

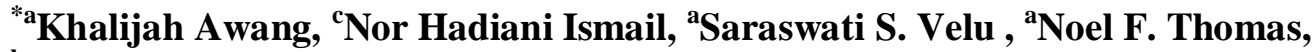 ${ }^{b}$ Jean-Frederic F. Weber and ${ }^{a}$ A. Hamid A. Hadi}

${ }^{a}$ Chemistry Department, Faculty of Science, University of Malaya, 50603 Kuala Lumpur, Malaysia.

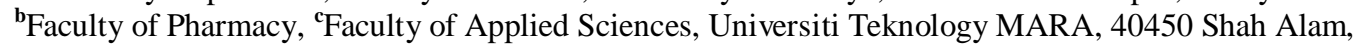
Selangor, Malaysia.

*Khalijah@um.edu.my (Corresponding author)

Received in $29^{\text {th }}$ Febuary 2008, accepted in revised form $20^{\text {th }}$ April 2009.

\begin{abstract}
A phytochemical study on Hunteria zeylanica Gard from the Malayan flora was conducted resulting in the isolation and characterization of six alkaloids. Five of them were indole alkaloids, which are eburnamine, isoeburnamine, eburnamenine, yohimbol and eburnamonine. In addition a venoterpene which contain a pyridine nucleus was also isolated. Structural elucidation of the compounds was accomplished by modern spectroscopic techniques: 2D NMR, IR, UV and MS.
\end{abstract}

ABSTRAK Hasil kajian fitokimia ke atas Hunteria zeylanica Gard. dari Malaysia yang diperolehi dari Terengganu, enam sebatian alkaloid telah berjaya dipencil dan dikenalpasti. Lima daripadanya adalah daripada jenis alkaloid indola, iaitu eburnamina, isoeburnamina, eburnaminina, yohimbol dan eburnamonina. Selain daripada itu satu sebatian venoterpena yang mengandungi nukleus piridina juga telah dipencilkan. Struktur semua sebatian telah ditentukan menggunakan kaedah spektroskopi: 2D RMN, IM, UV dan SJ.

(Keywords: Hunteria zeylanica Gard, eburnamine, isoeburnamine, eburnamenine, yohimbol, eburnamonine)

\section{INTRODUCTION}

Hunteria zeylanica Gard., a member of the Apocynaceae family, is native to tropical Africa and southeast Asia. In Malaysia, it can be found mostly in Pahang and Selangor [1]. However the plant sample used in this work was collected from Terengganu. The local name for Hunteria zeylanica is "kemuning hutan". Its latex has traditionally been used for smearing on sores caused by yaws. Among other traditional uses of $H$. zeylanica are for diarrhea, gastrointestinal discomfort and ulcers [2]. Pharmacologically, crude alkaloid extract of $H$. zeylanica leaves has been shown to produce byphasic effects on the central nervous system (CNS) causing depression and stimulation [3]. In an investigation of the crude alkaloid extract from the stem bark of the same plant on acute inflammatory responses, the results suggested that the inhibitory effect of the extract on acute edema formation are partly due to inhibition of 5-lipoxygenas and cyclooxygenase activity [4]. The extract inhibited leukotriene production by 5lipoxygenase in a concentration-dependent manner and blocked the production by $50 \%$ at $300 \mu \mathrm{g} / \mathrm{ml}$ concentration [5]. Previous studies by many groups on the chemical components on Hunteria showed that this genus is a rich source of monoterpenoid indole alkaloids [2] including alkaloids of medicinal values. Previous work on three varieties of $H$. zeylanica from, Kenya [2], Sri Lanka [6] and Thailand [7, 8] yielded many indole alkaloids, however, revealed differences in the alkaloidal components. Recently, we have communicated the structural elucidation of a new bisindole, huncaniterine, which possess a corynantheineaspidosperma type skeleton [9]. In continuation of our study on $H$. zeylanica, we wish to report in this communication our further findings.

\section{MATERIALS AND METHODS}

\section{Plant material}

The bark of Hunteria zeylanica was collected from Kampung Padang, Terengganu (Herbarium; KL 4345). Finely powdered bark was delipidated by overnight maceration using hexane. After removal of the hexane extract, the sample was then moistened with $5 \%$ $\mathrm{Na}_{2} \mathrm{CO}_{3}$. The moistened sample was then soaked in dichloromethane over a period of two weeks. The dichloromethane extract was drained daily and fresh dichloromethane was 
then added. The combined dichloromethane extract was evaporated under reduced pressure.

The crude extract was reconstituted in dichloromethane and the solution was extracted with $5 \%$ hydrochloric acid where the free bases are then converted to alkaloid salt. The dichloromethane layer was extracted until negative to Mayer's test. The combined acid extract was basified by addition of $\mathrm{NaOH}$ pellets. Basification of the aqueous phase converted the salts back to free bases, which was re-extracted with dichloromethane. The combined dichloromethane extract was dried over anhydrous sodium sulfate and concentrated under reduced pressure. The crude alkaloid was then subjected to various chromatographic procedures.

Isolation and purification of the alkaloids were carried out using different chromatographic techniques including column chromatography (CC) and preparative thin layer chromatography (PTLC). Six alkaloids were afforded: eburnamine 1(i-CC; EtOAc:Hexane: $\mathrm{MeOH}$; 6:4:0.4, ii-PTLC; EtOAc:Hexane: $\mathrm{MeOH} ; 6: 4: 0.4 ; \mathrm{R}_{\mathrm{f}}$ 0.55), isoeburnamine 2 (iCC; $\mathrm{CH}_{2} \mathrm{Cl}_{2}$ : MeOH:EtOAc; 9.5:0.6:0.2, iiPTLC; $\mathrm{CH}_{2} \mathrm{Cl}_{2}: \mathrm{MeOH}:$ EtOAc; 9.5:0.6:0.2; $\mathrm{R}_{\mathrm{f}}$ 0.27), eburnamenine 3 (i-CC; EtOAc: Hexane: $\mathrm{MeOH}$; 6:4:0.4, ii-PTLC; EtOAc:Hexane: $\mathrm{MeOH} ; 6: 4: 0.4 ; \mathrm{R}_{\mathrm{f}}$ 0.38), yohimbol 4 (i-CC; EtOAc: Hexane: $\mathrm{MeOH}$; 6:4:0.4, ii-PTLC; EtOAc: Hexane: $\mathrm{MeOH}$; 6:4:0.4; $\mathrm{R}_{\mathrm{f}}$ 0.36), eburnamonine 5 (i-CC; EtOAc: Hexane:MeOH; 6:4:0.2, ii-PTLC; EtOAc: Hexane:MeOH; 6:4:0.2; $\mathrm{R}_{\mathrm{f}}$ 0.49) and venoterpene 6 (i-CC; EtOAc: Hexane: $\mathrm{MeOH}$; 6:4:0.4, ii-PTLC; EtOAc: Hexane: $\mathrm{MeOH}$; 6:4:0.4; $\mathrm{R}_{\mathrm{f}}$ 0.25). Preliminary detection of the alkaloid components were done using Dragendorff's spray reagent and TLC is used for testing and selecting the right solvent systems for the chromatographic procedures. Structural elucidation of the isolated compounds was carried out using ${ }^{1} \mathrm{H}$ NMR, ${ }^{13} \mathrm{C}$ NMR, 2DNMR and mass spectroscopy.

\section{Eburnamine 1}

MS m/e: $296\left(\mathrm{M}^{+}\right), 278\left(\mathrm{M}^{+}-\mathrm{H}_{2} \mathrm{O}\right), 249\left(\mathrm{M}^{+}-\right.$ $\left.\mathrm{H}_{2} \mathrm{O}-\mathrm{C}_{2} \mathrm{H}_{5}\right), 208$.

UV $\lambda_{\max } n m(\log \varepsilon): 283$ (3.50), 229 (3.67).

IR $v \mathrm{~cm}^{-1}: 3317(\mathrm{OH})$.

${ }^{1} \mathrm{H}$ NMR $400 \mathrm{MHz}\left(\mathrm{CDCl}_{3}\right) \delta \mathrm{ppm}: 2.35-2.50$ (m, H-3) and 2.23-2.3 (m, H-3'), 3.17 (dd, $\mathrm{J}=14 \& 6, \mathrm{H}-5), 3.07$ (ddd, J=17,11\&6, H-5'), 2.83-3.00 (m, H-6), 2.35-2.50 (m, H-6'), 7.43 (dd, J=7\&2, H-9), 7.16-7.09 (m, H-10\&H-11), 7.72 (dd, J=7\&2, H-12), 1.66 (br qt, H-14), 1.24 (m, H-14'), 1.31 (d, J=14, H-15), 0.81 (td,
$\left.\mathrm{J}=13 \& 4, \quad \mathrm{H}-15^{\prime}\right), 5.52$ (dd, J=9\&5, H-16), 2.23-2.30 ( $\mathrm{m}, \mathrm{H}-17), 1.41-1.54$ (m, H-17'), 0.85 (t, J=7, H-18), 2.01 (dq, H-19), 1.41-1.45 (m, H-19'), 3.72 (s, H-21).

${ }^{13} \mathrm{C}$ NMR $100 \mathrm{MHz}\left(\mathrm{CDCl}_{3}\right) \delta \mathrm{ppm}: 132.4(\mathrm{C}-$ 2), 44.1 (C-3), 50.5 (C-5), 16.6 (C-6), 105.2 (C-7), 128.4 (C-8), 117.9 (C-9), 120.1 (C-10), 121.3 (C-11), 112.3 (C-12), 136.7 (C-13), 20.1 (C-14), 24.7 (C-15), 76.4 (C-16), 43.0 (C-17), 7.5 (C-18), 28.4 (C-19), 36.7 (C-20), 58.5 (C21).

\section{Isoeburnamine 2}

MS m/e: $296\left(\mathrm{M}^{+}\right), 278\left(\mathrm{M}^{+}-\mathrm{H}_{2} \mathrm{O}\right), 249\left(\mathrm{M}^{+}-\right.$ $\left.\mathrm{H}_{2} \mathrm{O}-\mathrm{C}_{2} \mathrm{H}_{5}\right), 208,179$.

UV $\lambda_{\max } \mathrm{nm}(\log \varepsilon): 283$ (3.80), 229 (4.40).

IR $v \mathrm{~cm}^{-1}: 3440(\mathrm{OH})$.

${ }^{1} \mathrm{H}$ NMR $400 \mathrm{MHz}\left(\mathrm{CDCl}_{3}\right) \delta \mathrm{ppm}: 2.50-2.75$ (m, 2H, H-3), 3.32 (dd, J=14\&6, H-5), 3.24 (ddd, J=17,11\&6, H-5'), 2.94-3.10 (m, H-6), 2.51-2.69 (m, H-6'), 7.40 (d, J=8, H-9), 7.117.19 (m, H-10\&H-11), 7.48 (d, J=7, H-12), 1.78 (br qt, $\mathrm{J}=13, \mathrm{H}-14$ ), 1.59-1.7 (td, J=13\&3, $2 \mathrm{H}, \mathrm{H}-15), 6.04$ (d, J=4, H-16), 1.96 (dd, $\mathrm{J}=15 \& 5, \mathrm{H}-17), 2.20-2.14$ (m, H-17'), 0.91 (t, $\mathrm{J}=7, \mathrm{H}-18$ ), 2.16 (dq, J=15\&7, H-19), 1.401.60 (m, H-19'), 3.84 (s, H-21).

${ }^{13} \mathrm{C}$ NMR $100 \mathrm{MHz}\left(\mathrm{CDCl}_{3}\right) \delta \mathrm{ppm}: 130.5(\mathrm{C}-$ 2), 44.8 (C-3), 51.3 (C-5), 16.7 (C-6), 105.2 (C-7), 128.7 (C-8), 118.3 (C-9), 120.0 (C-10), 121.2 (C-11), 110.1 (C-12), 134.9 (C-13), 20.7 (C-14), 26.2 (C-15), 74.3 (C-16), 40.0 (C-17), 7.5 (C-18), 28.9 (C-19), 34.6 (C-20), 59.3 (C21).

\section{Eburnamenine 3}

MS m/e: $278\left(\mathrm{M}^{+}\right), 249\left(\mathrm{M}^{+}-\mathrm{C}_{2} \mathrm{H}_{5}\right), 208,206$, 193.

UV $\lambda_{\max } \mathrm{nm}(\log \varepsilon): 312$ (3.40), 260 (3.80), 226 (3.90).

${ }^{1} \mathrm{H}$ NMR $400 \mathrm{MHz}\left(\mathrm{CDCl}_{3}\right) \delta \mathrm{ppm}: 2.68-2.71$ (m, H-3), 2.71-2.76 (m, H-3'), 3.34 (dd, $\mathrm{J}=14 \& 6, \mathrm{H}-5), 3.27$ (ddd, $\mathrm{J}=16,11 \& 5, \mathrm{H}-5$ '), 3.01 (m, H-6), 2.50 (ddd, J=17\&5, H-6'), 7.44 (d, J=8, H-9), 7.10 (t, J=7, H-10), 7.18 (t, J=7, $\mathrm{H}-11), 7.31$ (d, J=8, H-12), 1.64-1.74 (m, H14), 1.44-1.39 (m, H-14'), 1.47 (d, J=16, H-15), 1.13 (td, J=14\&4, H-15'), 6.90 (d, J=8, H-16), 5.06 (d, J=8, H-17), 0.98 (t, J=7, H-18), 1.98 (dq, J=14\&7, H-19), 1.74-1.64 (m, H-19'), 4.27 (s, H-21).

${ }^{13} \mathrm{C}$ NMR $100 \mathrm{MHz}\left(\mathrm{CDCl}_{3}\right) \delta \mathrm{ppm}: 130.0(\mathrm{C}-$ 2), 45.4 (C-3), 52.1 (C-5), 16.5 (C-6), 107.0 (C-7), 128.1 (C-8), 118.4 (C-9), 119.9 (C-10), 121.6 (C-11), 108.5 (C-12), 133.5 (C-13), 20.7 (C-14), 31.0 (C-15), 119.8 (C-16), 116.7 (C17), 8.9 (C-18), 27.5 (C-19), 37.3 (C-20), 55.9 (C-21). 


\section{Yohimbol 4}

MS m/e: $296\left(\mathrm{M}^{+}\right)$.

UV $\lambda_{\text {max }} \mathrm{nm}(\log \varepsilon): 2.83$ (3.50), 226 (4.13).

IR $v \mathrm{~cm}^{-1}: 3266(\mathrm{OH})$.

${ }^{1} \mathrm{H}$ NMR $400 \mathrm{MHz}\left(\mathrm{CDCl}_{3}\right) \delta \mathrm{ppm}: 3.20(\mathrm{~d}$, $\mathrm{J}=9.8$, H-3), 2.53 (bt, H-5), 3.03 (m, H-5'), 2.65 (bd, J=11.4, H-6), 2.86-3.02 (m, H-6'), 7.40 (d, J=7.7, H-9), 7.03 (t, J=7.4, H-10), 7.08 (t, J=7.2, H-11), 7.31 (d, J=7.9, H-12), 1.161.34 (m, H-14\&H-16), 1.71 (bt, J=15.1, H-14'), 1.50-1.58 (m, H-15), 1.59-1.66 (m, H-16'), 4.10 (m, H-17), 1.50-1.58 (m, H-18), 1.71 (bt, $\mathrm{J}=15.1, \mathrm{H}-18$ '), 1.34-1.50 (m, H-19), 1.34-1.50 (m, H-20), 2.06 (bt, H-21), 2.85 (d, J=8.3, H21').

${ }^{13} \mathrm{C}$ NMR $100 \mathrm{MHz}\left(\mathrm{CDCl}_{3}\right) \delta$ ppm: $134.8(\mathrm{C}-$ 2), 60.3 (C-3), 53.0 (C-5), 21.5 (C-6), 107.6 (C-7), 127.3 (C-8), 118.0 (C-9), 119.2 (C-10), 121.1 (C-11), 110.9 (C-12), 136.1 (C-13), 36.3 (C-14), 34.9 (C-15), 39.1 (C-16), 66.3 (C-17), 32.3 (C-18), 24.0 (C-19), 41.3 (C-20), 61.5 (C21).

\section{Eburnamonine 5}

MS m/e: $294\left(\mathrm{M}^{+}\right)$, 293, 265, 237, 224.

UV $\lambda_{\max } \mathrm{nm}(\log \varepsilon): 295$ (3.65), 267 (3.90), 242 (4.20), 207 (4.20).

IR $v \mathrm{~cm}^{-1}: 1703(\mathrm{C}=\mathrm{O})$.
${ }^{1} \mathrm{H}$ NMR $400 \mathrm{MHz}\left(\mathrm{CDCl}_{3}\right) \delta$ ppm: $2.37(\mathrm{~m}$, H-3), 2.59 (m, H-3'), 3.27 (m, H-5), 2.89 (m, H-6), 2.44 (m, H-6'), 7.41 (d, J=7, H-9), 7.30 (t, J=7, H-10), 7.28 (t, J=7, H-11), 8.34 (d, J=8, H-12), 1.72 (m, H-14), 1.37 (br d, H-14'), 1.47 (br d, J=14, H-15), 1.02 (td, J=14, H-15'), 2.59 (m, H-17), 2.63 (m, H-17'), 0.91 (t, J=8, H-18), 1.63 (m, H-19), 2.04 (m, H-19'), 3.97 (s, H-21). ${ }^{13} \mathrm{C}$ NMR $100 \mathrm{MHz}\left(\mathrm{CDCl}_{3}\right) \delta$ ppm: $134.2(\mathrm{C}-$ 2), 44.3 (C-3), 50.6 (C-5), 16.5 (C-6), 112.6 (C-7), 130.1 (C-8), 124.3 (C-9), 123.8 (C-10), 118.1 (C-11), 116.2 (C-12), 131.9 (C-13), 20.6 (C-14), 27.0 (C-15), 167.6 (C-16), 44.3 (C-17), 7.6 (C-18), 28.3 (C-19), 38.5 (C-20), 57.7 (C21).

\section{Venoterpene 6}

MS m/e: 149(M $\left.{ }^{+}\right), 134,130,120$.

UV $\lambda_{\max } n m(\log \varepsilon): 267$ (1.75), 209 (2.64).

IR $v \mathrm{~cm}^{-1}: 3224(\mathrm{OH})$.

${ }^{1} \mathrm{H}$ NMR $400 \mathrm{MHz}\left(\mathrm{CDCl}_{3}\right) \delta$ ppm: $8.3(\mathrm{~s}, \mathrm{H}-$ 2), 7.14 (d, J=5, H-5), 8.28 (d, H-6), 3.20 (dq, $\mathrm{J}=7 \& 13, \mathrm{H}-7), 4.45$ (td, J=5\&3, H-8), 3.08 (dd, $\mathrm{J}=5 \& 17, \mathrm{H}-9$ ), 2.90 (dd, J=2\&17, H-9'), 1.34 (d, J=7, $\mathrm{CH}_{3}$ ).

${ }^{13} \mathrm{C}$ NMR $100 \mathrm{MHz}\left(\mathrm{CDCl}_{3}\right) \delta \mathrm{ppm}: 147.4(\mathrm{C}-$ 2), 141.8 (C-3), 150.7 (C-4), 120.4 (C-5), 145.0 (C-6), 42.7 (C-7), 75.1 (C-8), 40.9 (C-9), $11.9\left(\mathrm{CH}_{3}\right)$.

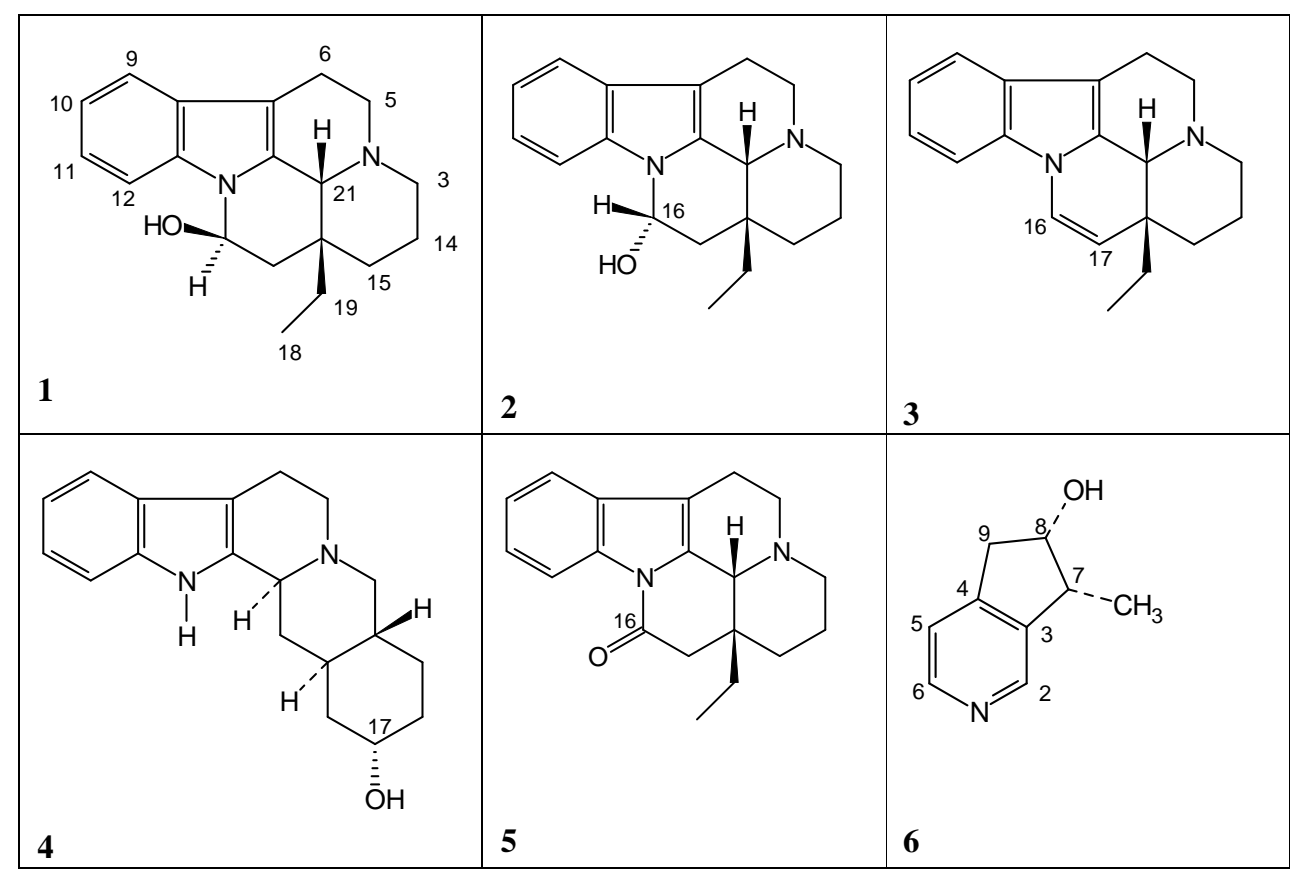

Figure 1. Alkaloids from Hunteria zeylanica Gard. 


\section{RESULTS AND DISCUSSION}

Five known indole alkaloids; eburnamine 1, isoeburnamine 2, eburnamenine $\mathbf{3}$, yohimbol $\mathbf{4}$, eburnamonine $\mathbf{5}$, and one pyridine type alkaloid; venoterpene 6 were isolated and identified.

Alkaloids 1-4 belong to the eburnane type while alkaloid $\mathbf{5}$ is of the yohimbine type. Identification and confirmation on the identity of these alkaloids were obtained by comparison of the spectral data $\left({ }^{1} \mathrm{H}\right.$ and ${ }^{13} \mathrm{C}$ NMR, IR, UV, MS) with the literature values [10-14].

Interestingly, eburnamonine $\mathbf{5}$ and venoterpene 6 has not been reported from the previous studies of $H$. zeylanica. In addition, the latter belong to the pyridine type alkaloid whereas all the other constituents isolated in this study and the previous ones belong to the indole family. This is also the first report on the occurrence of a pyridine type alkaloid (venoterpene 6) in the genus Hunteria. Therefore this study has shown that there were some significant differences in the chemical constituents of $H$. zeylanica collected from the Malayan Peninsular and the other parts of the world.

\section{ACKNOWLEDGEMENTS}

The authors wish to thank the Minister of Science and Technology for the financial support through the IRPA grant 09-02-01-0029.

\section{REFERENCE}

1. Whitmore, T. C. (1973). Tree Flora of Malaya - Volume Two. FRIM, Kuala Lumpur, Longman.

2. Lavaud, C., Massiot, G., Vercauteren, J., Men-Olivier, Le,L. (1982).Phytochemistry, 21,445-447.

3. Leewanich, P., Tohda, M., Matsumoto, K, Subhadhirasakul, S., Takayama, H., Aimi, N., Watanabe, H. (1996). Bio. Pharm. Bull., 19(3), 394-399.

4. Reanmongkol, W., Matsumoto, K, Watanabe, H., Subhadhirasakul, S., Takayama, H., Sakai, S. (1995). Bio. Pharm. Bull., 18(1), 33-36.

5. Reanmongkol, W., Matsumoto, K, Watanabe, H., Subhadhirasakul, S., Takayama, H., Sakai, S. (1995). Bio. Pharm. Bull., 18(6), 910-912.

6. Arambewela, L. S. R., Khuong-Huu, F. (1981). Phytochemistry, 20, 349-350.

7. Subhadhirasakul, , N., Takayama, H., Ponglux, D., Sakai, S. (1994). Chem. Pharm. Bull., 42, 991.

8. Takayama, H., Subhadhirasakul, S., Mizuki, J., Kitajima, M., Aimi, N., Pongluix, D., Sakai, S. (1994). Chem. Pharm. Bull., 42, 1957.

9. Khalit, M., Suzuki, T., Baba, Y., Zaima, K., Matsuno, Y., Hirasawa, Y., Awang, K., Hadi, A. H. A., Morita, H. (2007). Heterocycles, 74, 969.

10. Kam, T. S., Yoganathan, K., Chen Wei. (1996). Tetrahedron Lett., 37(20), 3603.

11. Feng, X. Z., Kan, C., Husson, H. P., Poter, P., Kan, S. K., Lounasma, M. (1984). J. Nat. Prod., 47, 117.

12. Torrenegra, R., Julio, A., Pedrozo, P., Achenbach, B., Baurei, P. (1988). Phytochemistry, 27(6), 1843.

13. Ray, A. B., Chatterjee, A. (1968). Tetrahedron Lett., 23, 2763-2766.

14. Bernard, W., Anton, R. (1994). J. Nat. Prod., 57, 287-290. 